\title{
MINERÍA DE HEMATITA EN LA COSTA SUR DEL PERÚ: INVESTIGACIONES ARQUEOLÓGICAS EN MINA PRIMAVERA*
}

\author{
HEMATITE MINING ON THE SOUTH COAST OF PERU: \\ ARCHAEOLOGICAL INVESTIGATIONS AT MINA PRIMAVERA
}

\author{
Kevin J. Vaughn ${ }^{1}$, Hendrik Van Gijseghem ${ }^{2}$, Moises Linares Grados ${ }^{3}$ y Jelmer W. Eerkens ${ }^{4}$
}

\begin{abstract}
A pesar que la minería es una actividad crítica para obtener materiales primas, se reconoce poco sobre la minería en los Andes prehispánicos. En este artículo presentamos evidencia de actividad minera antigua desde Mina Primavera, una mina antigua bien preservada de Nasca, costa sur de Perú, con fechas de explotación principalmente entre el periodo Intermedio Temprano (1-750 d.C.) y el Horizonte Medio (750-1.000 d.C.). Nuestras excavaciones proveen evidencia de extracción y proceso de hematita -usada en ofrendas y como pigmentos-, pero también evidencia de rituales que occurieron en la mina. Discutimos las excavaciones y nuestras interpretaciones de los artefactos y hallazgos que se han encontrado.
\end{abstract}

Palabras claves: minería, Nasca, Wari, hematita.

Despite the fact that mining is a critical activity to obtain raw materials, very little is known about mining in the prehispanic Andes. In this paper we present evidence of ancient mining activities from Mina Primavera, a well-preserved hematite mine from Nasca, south coast of Peru dating primarily to the Early Intermediate Period (ca. A.D. 1-750) and to the Middle Horizon (ca. A.D. 750-1,000). Our excavations provide evidence not only for the extraction and processing of hematite-used in offerings and as pigments- but also provide evidence for rituals that took place in the mine. We discuss excavations conducted within the mine over several seasons, as well as our interpretations of artifacts and features found.

Key words: Mining, Nasca, Wari, hematite.

La minería es una importante actividad en relación a la producción de bienes, es un elemento poco conocido dentro del desarrollo de organización social y político en la antigüedad (por ejemplo, ver Hanks y Doonan 2009:329; Maldonado y Rehren 2009; Nerantziz 2009). En los Andes, las evidencias de la minería antigua han sido escasas, primeramente porque la actividad minera prehispánica, colonial y moderna ha sido y es destructiva, elimina todo tipo de vestigio anterior (ver Eerkens et al. 2009). En efecto, la minería antigua en los Andes se entiende como uno de los elementos más importantes de la producción de bienes como metales, cerámicas, líticos y masonería de piedras (p.ej., Aldunate et al. 2008; Bird 1979; Burger y Matos 2002; Fuller 2004; Lechtman 1976; Núñez 2006; Petersen 1970; Salazar et al. 2010; Salazar et al. 2011; Shimada
1985, 1994, 1998; Stöllner 2009). En realidad, en los Andes prehispánicos la cultura material era dependiente de la minería extractiva de materias primas. Poco o casi nada se conoce acerca de la composición de comunidades mineras, y cómo las sociedades se organizaban en torno a la extracción y movimiento de los materiales extraídos es poco conocido por arqueólogos.

Mientras que Nasca y Wari son bien conocidos por sus tradiciones de producción cerámica muy elaborada (Proulx 2006; Menzel 1964), poco trabajo se ha realizado por conocer las materias primas de las cerámicas (ver Vaughn y Neff 2004). Nosotros hemos tratado de encontrar fuentes minerales para la producción de cerámica y hemos hallado unas minas en la región Nasca (Eerkens et al. 2009), incluyendo Mina Primavera, una mina prehispánica

* Artículo seleccionado del conjunto de ponencias presentadas en la Primera Reunión Internacional sobre Minería Prehispánica en América (PRIMPA), realizada en San Pedro de Atacama, Chile, diciembre 2010. Este manuscrito fue evaluado por investigadores externos y editado por Diego Salazar y Valentina Figueroa, en su calidad de editores invitados de la Revista.

1 Department of Anthropology, Purdue University, 700 West State Street, West Lafayette, IN 47907, USA. kjvaughn@ purdue.edu. 765-494-4700.

2 Department of Anthropology, Université de Montreal, Canada.

3 Arqueocare, Lima, Perú.

4 Department of Anthropology, University of California, Davis, USA. 
de hematita (Vaughn et al. 2007). En este artículo presentamos evidencias de actividad minera en Mina Primavera.

Hematita, más conocida como ocre, es un óxido de fierro $\left(\mathrm{Fe}_{2} \mathrm{O}_{3}\right)$, llamado en los Andes tacu o taco en quechua (Petersen 1970). Murúa (1925) nota cómo la gente en los Andes dio ofrendas de ocre rojo y harina de maíz al mar. Con mayor frecuencia, la hematita se utilizó como pigmento en los Andes, especialmente en Nasca (Petersen 2010:9; Vaughn et al. 2005; Yacovleff and Muelle 1934). La hematita se ha encontrado en cantidades pequeñas en Cahuachi (Kroeber y Collier 1998; Petersen 2010:9; Orefici y Drusini 2003), un sitio que aparentemente era un locus de producción cerámica (Orefici y Drusini 2003; Vaughn et al. 2006). La hematita se utilizó también como pigmento para textiles, objetos de madera, cosméticos y murales (Bonavia 1959, 1985; Phipps 1989; Yacovleff y Muelle 1934), y como pigmento para el cuerpo (Llagostera et al. 2000; Scalise y Di Prado 2006; Silva y Bahamondes 1969). A pesar de su importancia como elemento necesario en ofrendas y como pigmento, la explotación de este recurso no había sido entendida sino hasta hace poco (Salazar et al. 2011; Vaughn et al. 2007). Barba (1923:76) en su trabajo clásico de metalurgia en los Andes desestimó por completo la minería de hematita como actividad importante.

\section{Mina Primavera}

Mina Primavera se ubica al norte del Valle Ingenio en la Formación Portachuelo, compuesta por depósitos sedimentarios marinos del Cretáceo, con intervalos de piroclastos volcánicos intrusivos (Montoya et al.; Figura 1). Descubrimos Mina Primavera en el año 2004 gracias a la colaboración de informantes locales incluyendo mineros itinerantes. Es una cueva artificial; se establece sobre un promontorio rocoso empinado al frente de una mina moderna abandonada de baritina. En la mina, al interior de su techo presenta una coloración roja con huellas de martillos y un piso cubierto de desechos de piedras. La cueva mide aproximadamente 20 por 30 metros, con una altura media de dos metros. El piso de la mina baja unos metros al sur y al oeste para formar tres galerías separadas (Figura 2).

Galería 1 (G1) es la galería principal, la más accesible. La mayoría de las excavaciones se han llevado a cabo en este sector. La galería tiene aproximadamente $100 \mathrm{~m}^{2}$ y se inclina suavemente al sur hacia la Galería 2 y al oeste a la Galería 3. Siete morteros ubicados en el suelo sirvieron para la trituración inicial de hematita localizados en la Galería 1. La Galería 2 presenta un acceso difícil por ser muy estrecha, después se abre para formar un espacio de $150 \mathrm{~m}^{2}$, aunque es difícil evaluar el tamaño preciso por la gran cantidad de desechos minerales cubriendo el piso. Encontramos once morteros en la superficie de esta galería. En la Galería 3 no se encontró ningún mortero, quizás porque se hallan cubiertos por cascajos. Además de las tres galerías, se encuentra una cámara pequeña (2x2 $\mathrm{m})$ accesible por la Galería 3.

Se han excavado $40 \mathrm{~m}^{2}$ en Mina Primavera a lo largo de tres temporadas en 2004, 2005 (Vaughn et al. 2007) y 2010. Estas excavaciones han revelado que la mina fue explotada por más de un milenio. Se logró identificar que aparte de la explotación de hematita, encampamiento en la mina, se realizaron actividades rituales como tocar música. Ahora, pasamos a una descripción de las excavaciones en la Mina Primavera.

Dos estratos principales fueron registrados en las excavaciones. El primer estrato se compone de sedimentos de origen eólico que representa el uso posterior a la explotación minera (Capa A). El otro estrato se compone de desechos de actividad minera mezclados con pequeños cascajos que representa la explotación principal de la mina (Capa B). Los artefactos incluyen cerámicas, textiles, restos botánicos y fauna, también herramientas de piedras, maderas y artefactos de uso ceremonial como Spondylus e instrumentos musicales. Con la excepción de los instrumentos musicales, todos los artefactos fueron encontrados fuera de contexto.

Los fragmentos de cerámica diagnóstica incluyen desde fragmentos del Formativo hasta el periodo Intermedio Tardío (Tabla 1). Un fragmento formativo y otro del periodo Intermedio Tardío fueron encontrados en la Galería 1, pero en Capa A (Figura 3) sugiere prospección después de la explotación principal. A pesar de esta distribución de cerámicas, la mayoría son del periodo Intermedio Temprano, encontradas en las tres galerías, pertenecen a la fase Nasca Temprano (1-450 d.C.: ver Vaughn 2009). En conjunto, la cantidad de cerámica Nasca sugiere que la explotación durante el periodo Intermedio Temprano fue más persistente en antigüedad. Adicionalmente, recuperamos evidencia de explotación Wari en Galería 1, incluyendo un 
fragmento de nariz de una jarra de cerámica Wari, que comúnmente se piensa que se trata de una vasija de élite Wari (Cook 1984-1985), hallado en la entrada a la mina.

Siete fechados de carbono catorce sugieren que las fechas de explotación eran aproximadamente 1-1.400 d.C. (Tabla 2). La Capa A presenta fechas que se agrupan desde d.C. 900-1.400, mientras que la Capa B tiene fechados ubicados en el primer milenio d.C. Los fechados establecidos en Capa B sugieren que la explotación principal en la mina fue durante la época Nasca Temprano.

La mayoría de los textiles eran fragmentos de bolsas de algodón en urdimbre simple, tejido que es típico de telar de cintura de los Andes desde 800 a.C. La mayoría de los fragmentos textiles presenta huellas de rasgado, indicando claramente que se usaron para el transporte del mineral. También recuperamos grupos de cuerda, un fragmento lana de tejido Nasca Temprano y una honda (Figura 4), la cual fue encontrada en la galería 1, Capa A. Por lo tanto, la honda probablemente represente una prospección postexplotación. Se han registrado también restos botánicos como maíz y botella de calabaza, así como una pequeña cantidad de pacay, lúcuma y achupalla. Se recuperó una cantidad pequeña de maní que fue asociado a la nariz de jarra Wari.

Las excavaciones revelaron numerosas herramientas con relación a la actividad minera en Mina Primavera. Herramientas de piedra que se hicieron de una limolita ${ }^{1}$ se encuentran fuera de la mina. Las herramientas se clasifican en cinco tipos: (1) Lajas usadas como pala, (2) cuñascinceles de columna, (3) martillos de piedras de mano, (4) hachas de piedra semicircular a triangular con mango de madera y (5) cantos rodados (a menudo con pulimento) utilizados como herramientas de pulido (Figura 5). También se recuperaron algunas herramientas de madera, probablemente utilizadas como cuñas en la minería. Las herramientas de minería se parecen mucho a las que se encuentran con el "Hombre de Cobre" de la Mina Chuquicamata, Chile (Bird 1979). Este

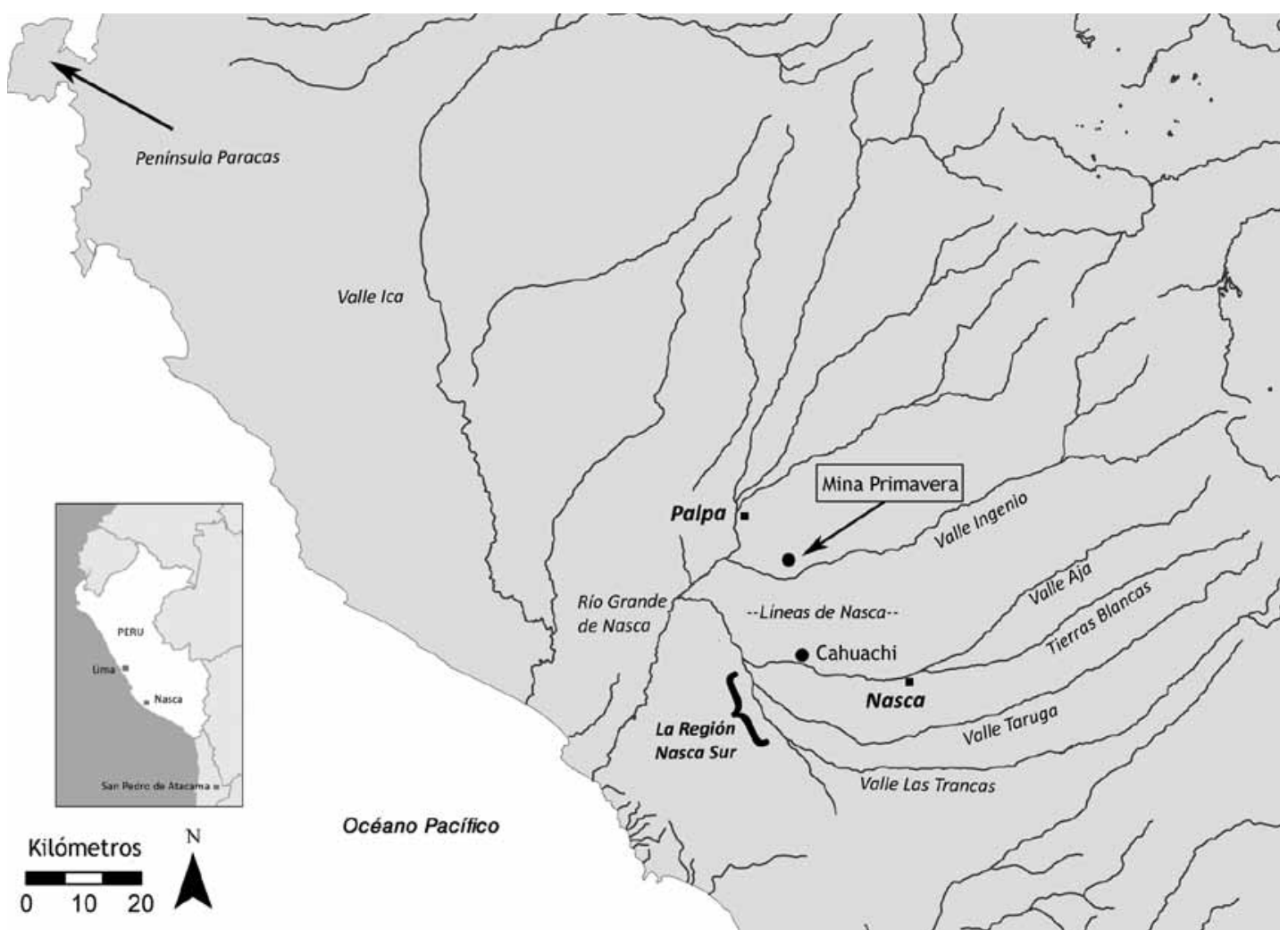

Figura 1. Mapa de la costa sur del Perú. Se marcan los sitios mencionados en el texto.

Map of the south coast of Peru. Sites mentioned in text are noted. 


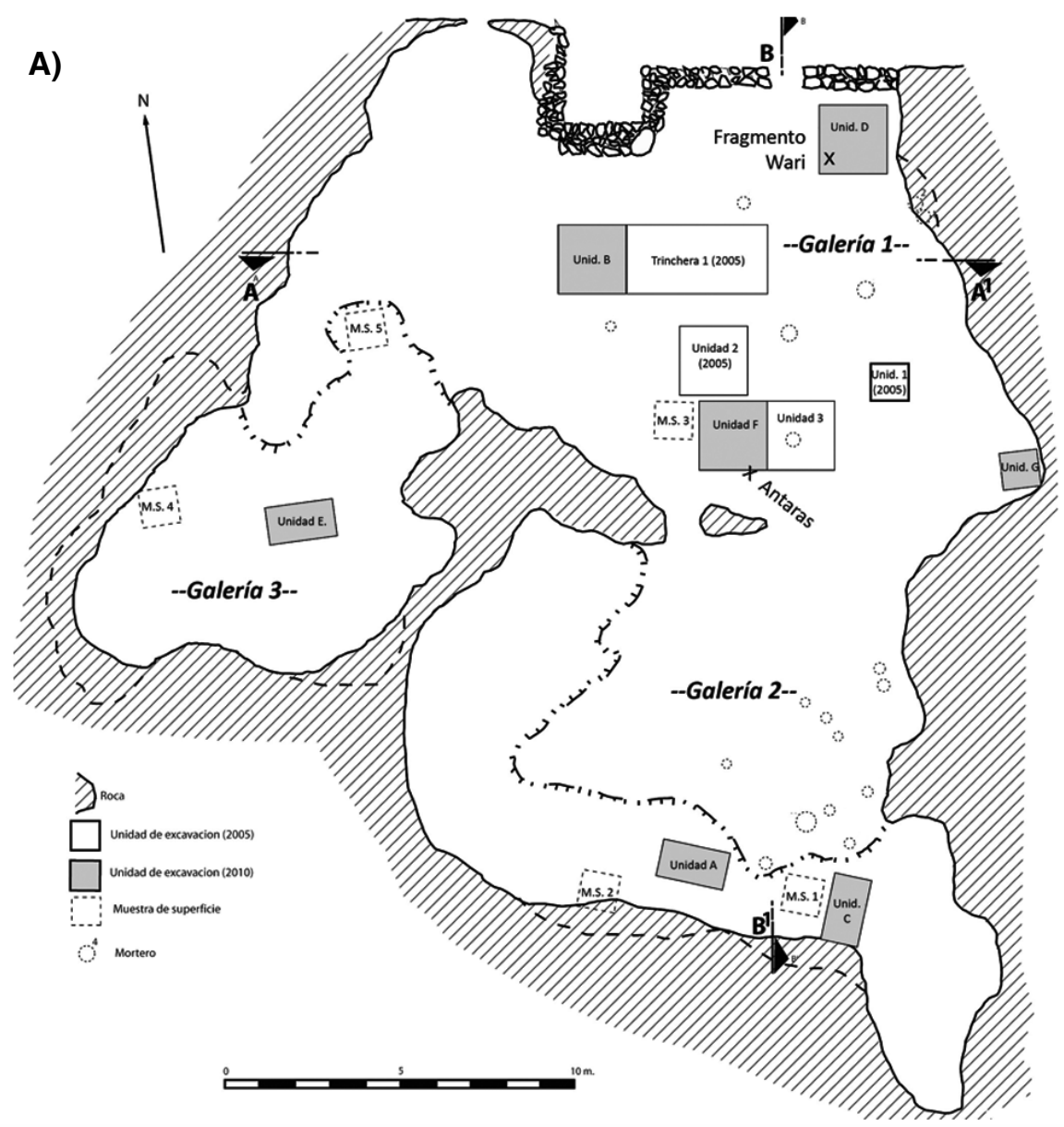

B)

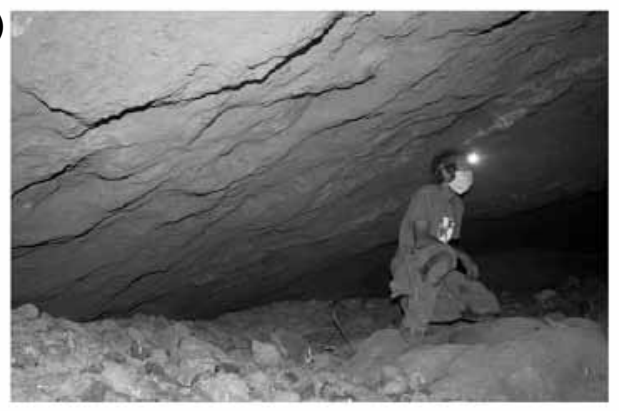

C)

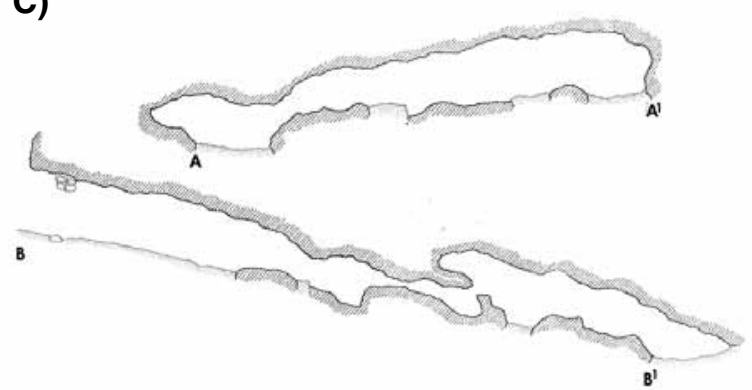

Figura 2. (A) Plano de Mina Primavera. (B) Al interior de la mina, galería 2. (C) Perfiles de la mina con la misma escala que (A). (A) Plan of Mina Primavera. (B) Interior of the mine, gallery 2. (C) Profiles of the mine. Scale is the same as in (A).

gran parecido de las herramientas de minería a miles de kilómetros de distancia se ha observado en otros contextos de minería prehispánica (ver, p.ej., Salazar et al. 2011; Shimada 1994), lo que sugiere que esta "caja de herramientas" fue común a través de los Andes en la antigüedad.

Fue poca la presencia de restos animales; la mayor evidencia se registró en la Capa A y fue asociada con la actividad natural de estos pequeños animales (por ejemplo huesos de aves y ratones). Se encontraron moluscos, incluyendo Spondylus (Figura 4), bien conocidos como símbolo de agua y fertilidad en los Andes, procedentes de la Capa B, Galería 1.

En la Galería 1 también se recuperó un par de antaras de caña intacta (Unidad F, Capa B) y 
fragmentos de una flauta de hueso (Figura 4). Las antaras son iguales, cada una presenta siete tubos de caña que van disminuyendo de tamaño y atadas con hilo de algodón. El tubo más largo de cada par está envuelto en hilo de algodón de color marrón con un diseño no diagnóstico. El centro de cinco tubos de cada par tiene discos de mate (véase Figura 4.D) de enchufar la base del tubo para que pudiera ser tocado. A pesar de que las antaras son frágiles se encuentran en perfecto estado de conservación, interpretando que fueron cuidadosamente depositadas y que se encuentran en un contexto primario (el único contexto primario recuperado hasta ahora en las excavaciones). Un disco de mate de un tubo se sometió a la datación AMS (Universidad de Arizona)

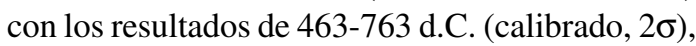
con estos datos podemos ubicarlo en el periodo Intermedio Temprano u Horizonte Medio. Debido a este hallazgo sospechamos que en las áreas que aún quedan pendientes por excavar podemos lograr mayores descubrimientos de contextos asociados estrictamente a la actividad minera de hematita.

Tabla 1. Cronología de la región Nasca Sur y Palpa. Mina Primavera localizada en la frontera entre las dos zonas. Chronology for the Southern Nasca Region and Palpa. Mina Primavera is located in the boundary between these two zones.

\begin{tabular}{|c|c|c|c|c|}
\hline Horizontes / Periodos & Periodo Local & Cultura & $\begin{array}{l}\text { Fecha aproximada en la } \\
\text { región Nasca Sur } \\
\text { (Schreiber y Lancho 2006) }\end{array}$ & $\begin{array}{l}\text { Fecha aproximada en } \\
\text { Palpa (Reindel 2009; } \\
\text { Unkel et al. 2007) }\end{array}$ \\
\hline Horizonte Tardío & -Inca- & Inca & 1.476-1.532 d.C. & $1.400-1.532$ d.C. \\
\hline Periodo Intermedio Tardío & - Tiza- & Tiza & 1.000-1.476 d.C. & 1.000-1.400 d.C. \\
\hline Horizonte Medio & -Loro- & Loro, Wari & 750-1.000 d.C. & 620-1.000 d.C. \\
\hline \multirow[t]{3}{*}{ Periodo Intermedio Temprano } & $-N a s c a-$ & Nasca Tardío & 550-750 d.C. & 440-620 d.C. \\
\hline & & Nasca Medio & 450-550 d.C. & 325-440 d.C. \\
\hline & & Nasca Temprano & 1-450 d.C. & 90-325 d.C. \\
\hline \multirow[t]{2}{*}{ Horizonte Temprano } & -Formativo- & Proto Nasca (Nasca Inicial) & 100 a.C. -1 d.C. & 200 a.C.-90 d.C. \\
\hline & & Paracas & 800-100 a.C. & $800-200$ a.C. \\
\hline
\end{tabular}
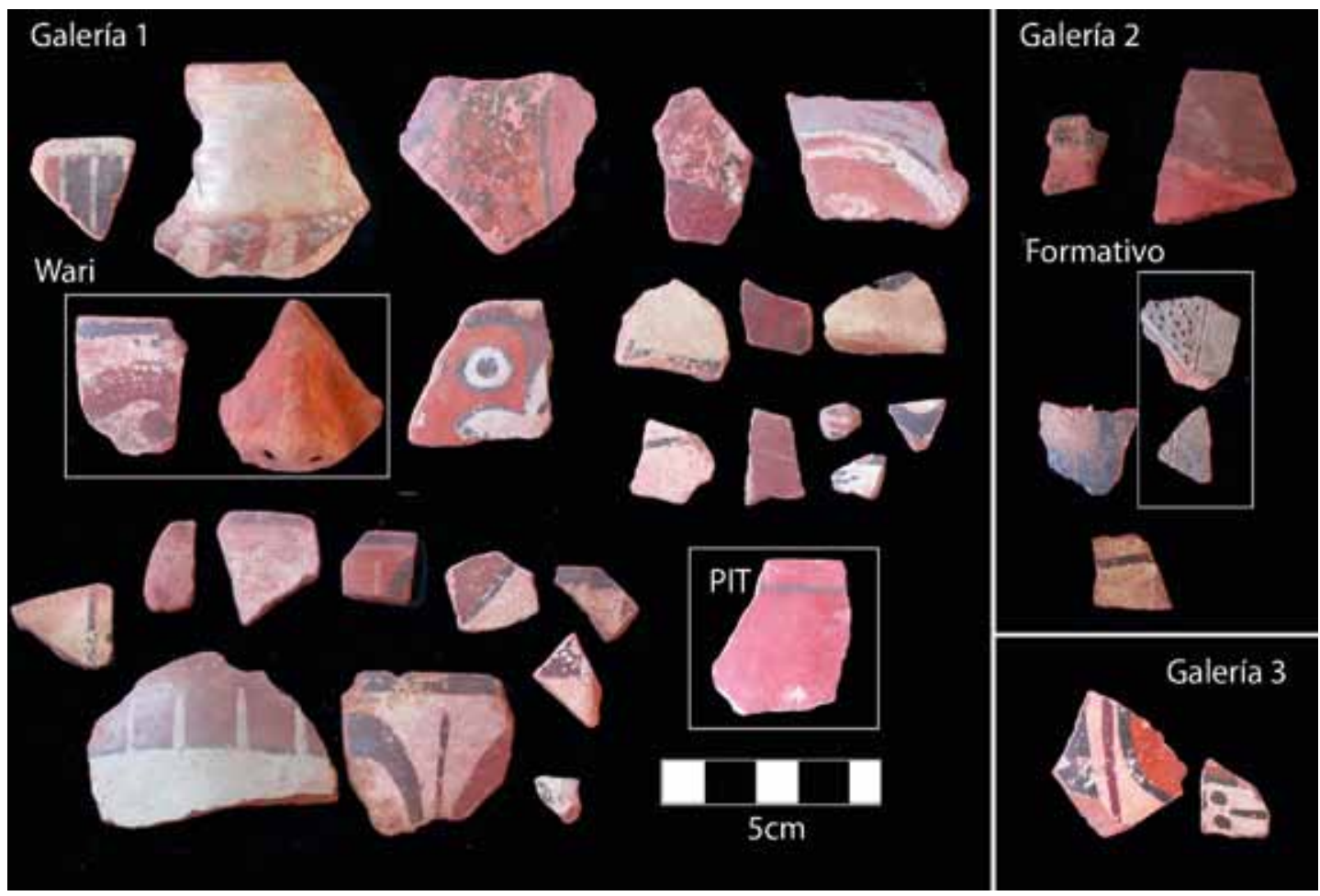

Figura 3. Cerámicas de las tres galerías en Mina Primavera. La mayoría de las cerámicas pertenecen al periodo Nasca. Ceramics from the three galleries in Mina Primavera. The majority of the ceramics are from the Nasca Period. 
Tabla 2. Fechados de radiocarbon desde Mina Primavera. Se han calibrado por INTCAL 09 (Reimer et al. 2009). Radiocarbon dates from Mina Primavera calibrated using INTCAL 09 (Reimer et al. 2009).

\begin{tabular}{cccccrc}
\hline \# de Muestra & Galería & Unidad & Capa & Material & Fecha C14 & Años calibrado $2 \sigma$ \\
\hline AA72022 & 1 & 2 & A & Maíz & $505 \pm 43$ & $1.316-1.455$ d.C. \\
Beta 195717 & 1 & 1 & A & Maíz & $990 \pm 70$ & $896-1.210$ d.C. \\
AA72023 & 1 & 2 & B & Maíz & $1.901 \pm 44$ & $17-230$ d.C. \\
AA72024 & 1 & 3 & B (A2) & Maíz & $1.951 \pm 35$ & 38 a.C.-126 d.C. \\
AA72021 & 1 & T1 & B & Maíz & $1.961 \pm 43$ & 48 a.C.-128 d.C. \\
AA94038 & 3 & E & B (D) & Mate & $1.832 \pm 35$ & $83-311$ d.C. \\
AA94039 & 2 & C & B & Mate & $1.722 \pm 35$ & $241-403$ d.C. \\
AA91681 & 1 & F & B & Tapa de antara & $1.480 \pm 55$ & $463-763$ d.C. \\
\hline
\end{tabular}

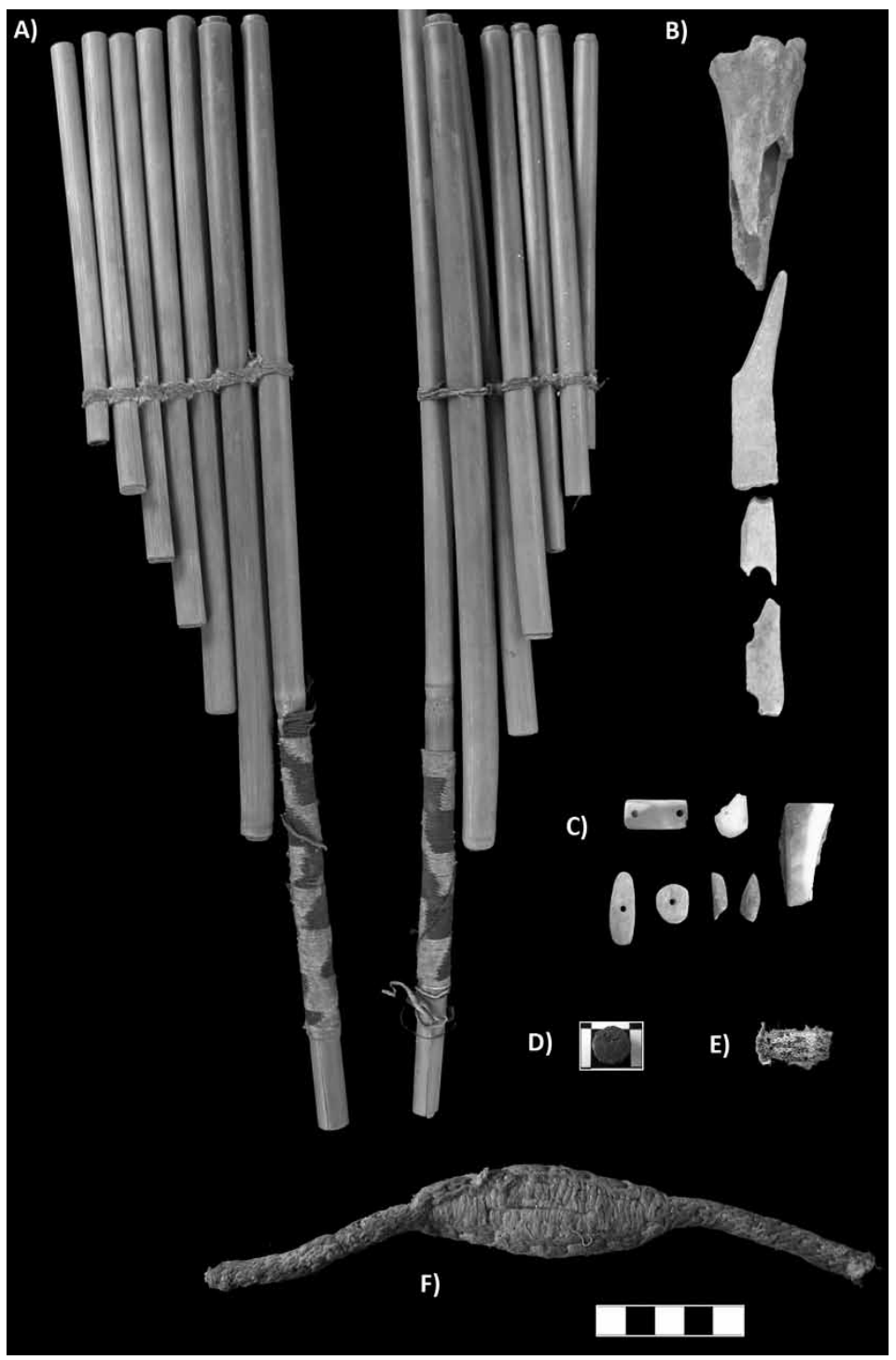

Figura 4. Artefactos de Galería 1 Mina Primavera. (A) Par de antaras. (B) Fragmentos de una flauta de hueso. (C) Fragmentos de Spondylus (modificados y sin modificar). (D) Un ejemplo de disco de mate de enchufar la base del tubo de antaras. (E) Un fragmento lana de tejido Nasca Temprano. (F) Honda, se encuentra en Capa A.

Artifacts from Gallery 1. (A) Pair of panpie. (B) Fragments ofa bone flute. (C) Fragments of Spondylus (modified and unmodified). (D) An example of a gourd plug from the base of a panpie. (E) Fragment of Early Nasca wool tab. (F) Sling found in Capa A. 


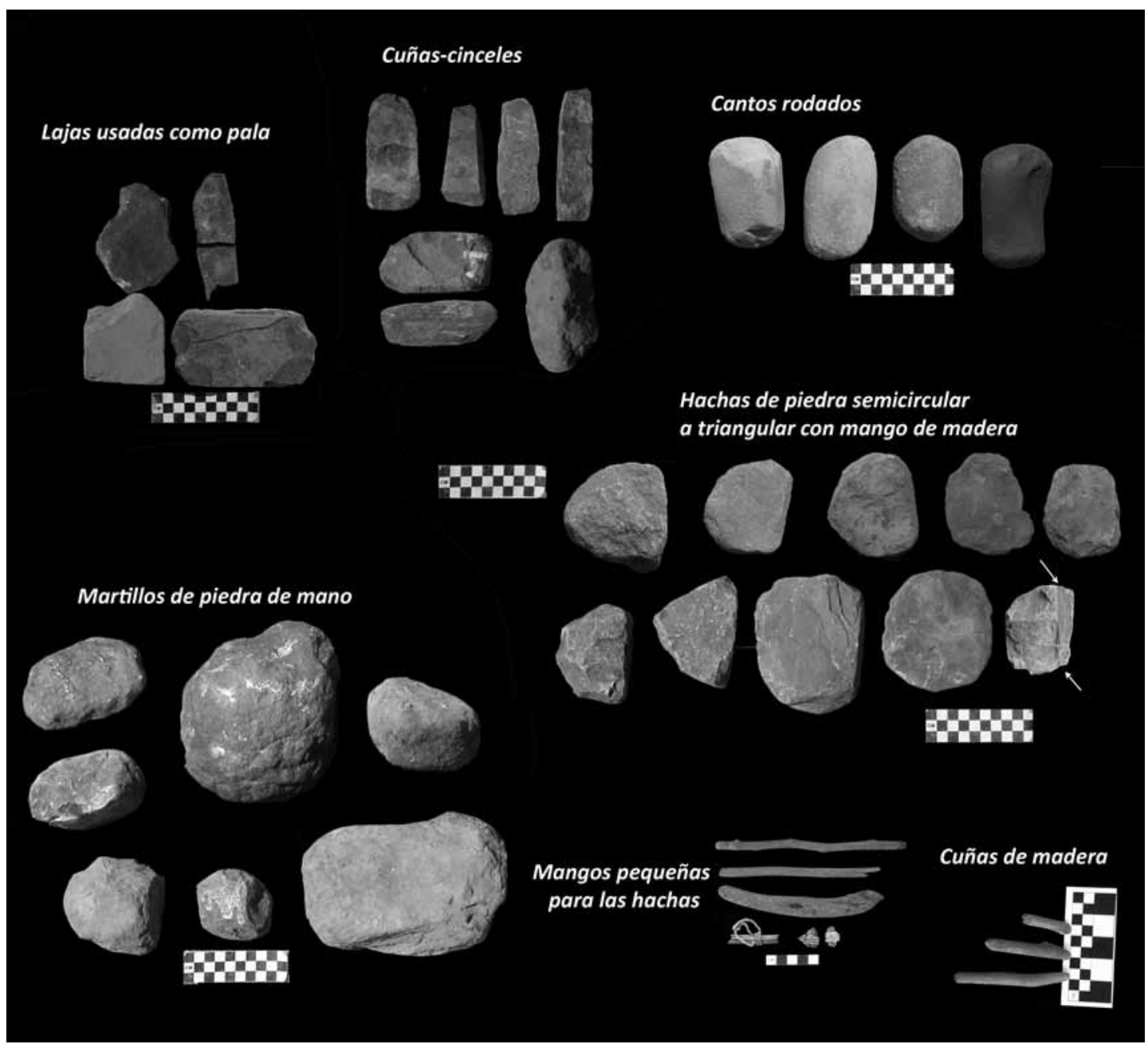

Figura 5. Herramientas de piedra y madera de la Mina Primavera. Las herramientas se han usado para sacar y procesar hematita. Stone and wooden tools from Mina Primavera. Tools were used to extract and process hematite.

\section{Discusión}

Nuestros resultados revelan evidencia importante sobre la organización minera en la región Nasca. Los artefactos y hallazgos en Mina Primavera sugieren que por lo menos hace 2.000 años los mineros traían alimentos, textiles para transporte, madera para herramientas, así como ofrendas y artefactos rituales para ofrendar a la mina. La basura doméstica en las excavaciones sugiere que los mineros probablemente acamparon en el lugar. Hemos encontrado asentamientos temporales consistentes en pequeñas terrazas asociadas a martillos de piedra, desechos líticos y cuencos rotos. Estos campamentos fueron utilizados para planificar las actividades mineras en la región, son muy pequeños en comparación con otros campamentos mineros que han sido investigados en valles cercanos (Eerkens et al. 2009).

Por lo tanto, creemos que la mayoría de las actividades mineras, básicamente en la extracción y embalaje, se desarrolló ahí mismo dentro de la Mina Primavera. Mientras que los mineros acampaban en la mina, extraían piedras nativas para ser usadas como herramientas, elaboradas con limolita y con madera. Se molió la hematita en morteros localizados en el suelo de la mina, y transportaron el mineral molido en bolsas textiles sin decoración a una ubicación externa donde lo procesaron seguramente con mayor especialización para ser usado como pigmento.

La Mina Primavera presenta una gran extensión sugiriendo que la escala de operaciones mineras 
prehispánica era intensa. Basado en el tamaño de la mina, estimamos que casi $700 \mathrm{~m}^{3}$ (aproximadamente 3.710 toneladas en $5.3 \mathrm{~g} / \mathrm{cc}$ ) de hematita han sido extraídos a lo largo de dos milenios para crear la cueva. Si asumimos nuestros fechados radiocarbónicos tenemos que fue utilizada unos 1.400 años; un medio metro cúbico (2,65 toneladas métricas) de hematita fue extraída por año. Pero la evidencia sugiere que la extracción fue mayor durante algunos periodos, especialmente Nasca Temprano. Si asumimos un lapso de 400 años, el ritmo de extracción fue más de 1,75 metros cúbicos por año. De cualquier manera, es una cantidad sustancial de hematita extraída de la mina.

También encontramos evidencia de explotación en el Formativo a partir de la presencia de un fragmento de cerámica. Dado que cerámicas y textiles pintados fueron importantes para la cultura Paracas, no es una gran sorpresa. Lo curioso, sin embargo, es que el fragmento formativo fue encontrado al fondo de la mina donde se habría desarrollado la explotación más tardía. Pero dado que la estratigrafía horizontal en la mina no se logra entender totalmente (esperamos más AMS fechados de todos los contextos excavados), ya que todos los contextos son disturbados fuertemente por actividades mineras, este fragmento claramente no se encuentra en un contexto primario.

El descubrimiento de un par de antaras y los fragmentos de una flauta de hueso animal demuestran que actividades dentro de la mina incluyeron el tocar música. Por supuesto, evidencias etnográfica y etnohistórica sugieren que en las minas andinas la música tenía importancia simbólica significativa. Por ejemplo, Cobo (1890-1895) sugirió que algunas minas fueron huacas que la gente de los Andes le rezaba para ceder su metal. Además, en su trabajo Nash (1979) informó que los rituales y ceremonias andinas que acompañaban a la minería en Bolivia continuaban en la actualidad a pesar de siglos de desplazamiento forzado y los esfuerzos coloniales para disipar los viejos sistemas de creencias. Estos rituales incluyeron baile, música y ofrendas a una figura que se conoce como "Supay", o el "diablo", de la mina (véase también Gisbert 2010).

Dada esta evidencia, interpretamos que las antaras se utilizaron en una ceremonia ritual asociada a la actividad minera. Esta interpretación es compatible con nuestro descubrimiento de fragmentos de Spondylus de Ecuador, frecuentemente usado en rituales andinos (Paulsen 1974). Tenemos que ser cautelosos en la reconstrucción de este ritual; sin embargo, los datos etnográficos y etnohistóricos son ricos en información para aclarar la importancia de las minas en el paisaje andino prehispánico. Proponemos la hipótesis de que los rituales fueron posiblemente para que los espíritus de los cerros y de la mina-como Supay-cedieran generosamente sus minerales.

Las antaras se encontraron tardíamente, no tuvimos la oportunidad excavar más cerca a su ubicación. Como se mencionó anteriormente, estos artefactos fueron los únicos que estuvieron en contexto primario. Esto indicaba que las antaras que fueron colocadas allí deliberadamente-quizás como una ofrenda- o asociadas con artefactos adicionales de un contexto primario - posiblemente a un entierro. Se requiere trabajo adicional para probar esta inferencia.

La fecha radiocarbónica (d.C. 463-763, cal. $2 \sigma$ ) de las antaras las coloca en fases tardías de la secuencia Nasca, o parte del Horizonte Medio Temprano. Reindel (2009) propone que en la región Palpa el Horizonte Medio empieza a 600 d.C. basado en numerosos fechados de radiocarbono (Unkel et al. 2007). Vaughn (2010) ha sugerido que las diferencias entre las fechas entre la región Nasca sur donde el comienzo de Horizonte Medio es normalmente 750 d.C. (ver Schreiber y Lancho Rojas 2006) y la región Palpa puede ser una consecuencia de la dinámica regional del poder, empezando en Nasca Medio (fases 4 y 5 en la secuencia de Palpa). Mina Primavera se ha localizado en una frontera entre estas subregiones de la costa sur, pero los fechados son más afines a la secuencia Palpa. Entonces, sugerimos que las antaras son del Horizonte Medio.

Por ahora, se necesitará trabajar más para determinar con exactitud si las antaras pertenecen a la cultura Nasca o al Horizonte Medio. Para nuestro conocimiento, no hay un ejemplo de antaras de caña que se haya encontrado o fechado para el periodo Nasca. Todas las antaras Nasca halladas han sido de cerámica, la mayoría se hicieron por engobe de fundición (Dawson 1964). Haeberli (1979) ha sugerido que todas las antaras en Nasca se hicieron de cerámica y no fue sino hasta la última parte del período Intermedio Temprano y dentro del Horizonte Medio que la gente de la costa empezó a elaborar antaras de caña. Por lo tanto, tenemos razones para creer que las antaras datarían en el Horizonte Medio.

Sugerimos previamente que la minería llevada a cabo en Mina Primavera durante los tiempos Nasca 
podría seguir siendo explotada parcial e informal, tal vez por mineros itinerantes (Eerkens et al. 2009; Vaughn et al. 2007). Todavía creemos que la explotación entera de la mina durante unos siglos no habría sido requerida a tiempo completo; sin embargo, fechados adicionales de Mina Primavera son necesarios para evaluar completo el carácter de la minería en el sitio.

Nuestras excavaciones han revelado que los Wari explotaron el lugar más tiempo de lo que se pensaba, probablemente no utilizaron la mina tan frecuentemente como los Nasca, creemos que el carácter y organización de la minería podría haber cambiado con la presencia Wari. Específicamente, el fragmento de la jarra de élite cerca de la entrada de la mina asociada directamente con una cantidad pequeña de maní (la única evidencia de este alimento vegetal) podría indicar supervisión de élites.

Es bien conocido que los Incas supervisaban las actividades mineras, la evidencia arqueológica de esto es relativamente escasa. Para la verificación de una jornada completa, usualmente se requieren facilidades permanentes para apoyar a supervisores y administradores (p.ej., Salazar et al. 2010). No hay ninguna evidencia de estas facilidades en Mina Primavera. Sin embargo, no hay razón para creer que supervisión se requiere porque había variedades considerables en la cantidad y tipo de supervisión en medio de los incas (ver Berthelot 1986: 75-76).

Además, si las minas tenían un significado simbólico en la antigüedad como la tuvieron en época contemporánea e inca, sospechamos que los mineros llevaron a cabo rituales en estas minas, así la minería haya sido en pequeña escala o patrocinada por el Estado. Mientras que los imperios se apropiaban de los recursos, también se apropiaban de los rituales, paisajes sagrados y el capital simbólico (Schreiber 2005a). Entonces, el interés de Wari en la Mina Primavera no puede haber sido sólo la hematita, sino la minax misma como parte del paisaje sagrado de Nasca (ver Schreiber 2005b).

\section{Conclusiones}

Si hubo un cambio en la estructura de la minería entre el periodo Intermedio Temprano y el Horizonte Medio, no sería sorpresa. Es bien conocido que cerámicas decoradas finas tenían un papel importante en la sociedad Nasca (p.ej., ver Proulx 2006; Vaughn 2009) y Mina Primavera fue claramente explotada por mineros Nasca. Nasca no se organizó como un Estado (ver Reindel 2009); sin embargo, el poder de sus líderes se basó en su participación en ceremonias religiosas y no en la administración de actividades económicas (Vaughn 2010). En el Horizonte Medio, la cerámica pintada fina continuaba siendo importante para los Wari. Además, mantuvieron muchos aspectos tecnológicos e iconográficos de la cerámica Nasca (Menzel 1964). No es sorprendente que el imperio Wari haya tomado gran interés en una fuente de hematita de buena calidad como la disponible en la Mina Primavera.

No obstante, consideramos nuestras sugerencias como hipótesis que se deben probar en el futuro. Se necesita mucho trabajo en Mina Primavera, y esperamos ubicar nuevas minas en la región. Por ahora nos gustaría concluir con unas preguntas que puedan ser abordadas por nuestra continua investigación en la región:

Primero, ¿cómo se organizaba en la costa sur del Perú en los tiempos prehispánicos y específicamente saber cómo se desarrolló el cambio entre la minería de periodo Intermedio Temprano y el Horizonte Medio? Nuestras expectativas teóricas de imperialismo responden a unas evidencias que tenemos en Mina Primavera que nos permiten sugerir esto. Pero se necesita más trabajo todavía. Si un imperio coopta por los recursos y paisajes sagrados, es curioso que no haya evidencia de explotación de la Mina Primavera por los incas, quienes poblaron la región Nasca durante el Horizonte Tardío, por alguna razón no la incluyeron como su paisaje sagrado (Schreiber 2005b). Además, que la explotación de la mina durante el periodo Intermedio Tardío parece estar confinada a alguna limitada prospección. Por el contrario, nuestro estudio en Ica dirigido por Van Gijseghem mostró un gran aumento en la minería en el período Intermedio Tardío. Sugerimos que la hematita de alta calidad en Mina Primavera se había agotado para el periodo Intermedio Tardío, o quizás no era tan importante como recurso, como sucedió en los periodos más tempranos. Nuestro trabajo previo (Vaughn et al. 2005) sugirió que se usó hematita en menor cantidad para la alfarería como pigmento en el Periodo Intermedio Tardío, quizás representaba la falta de interés en este recurso.

Segundo, ¿qué tipo de actividades se desarrollaron en las minas? La primera vez que registramos Mina Primavera y comenzamos a excavarla, inferimos algunas de las actividades que tienen lugar en la mina, incluyendo la extracción y el procesamiento de hematita. Los descubrimientos del 2010 
han contribuido a entender que la actividad minera es mucho más compleja, al hallarse contextos de instrumentos musicales en el lugar. Nuestras interpretaciones siempre se verán obstaculizadas por el limitado registro arqueológico -especialmente al interior del contexto de las minas-, pero nos sentimos alentados por estos hallazgos recientes en comparación con el rico registro etnográfico y etnohistórico de la minería indígena en los Andes.

Tercero, queremos poner fin a la especulación para qué la hematita de Mina Primavera fue utilizada. Hemos aludido al hecho de que la hematita se utilizó en los pigmentos, especialmente para la pintura en la cerámica Nasca y Wari. Por ahora, lamentablemente, no tenemos ninguna evidencia directa que demuestre que la hematita de esta mina fue utilizada en realidad para pintar la cerámica. Recientemente, Eerkens ha empezado a encontrar hematita de la región usando proporciones de isótopos de hierro (Eerkens et al. s.f.). El método ha distinguido fuentes diferentes de la región incluyendo Mina Primavera. Usando la misma técnica para una muestra pequeña de cerámicas, no hemos sido capaces de igualar las muestras de pigmento con muestras de Mina Primavera, sugiriendo que por lo menos en las muestras de siete fragmentos de la región Nasca Sur, hematita de Mina Primavera no se usó para pigmentos. Sin embargo, descubrimos que la hematita de Mina Primavera es casi idéntica con una muestra pequeña de pigmento hematita colectado por Kroeber, en la década de 1920, de Cahuachi y actualmente localizado en el Museo Field en Chicago. Es un poco temprano para afirmar que este descubrimiento vincula directamente a Mina Primavera con las actividades que tienen lugar en Cahuachi, que incluía rituales, la peregrinación y la producción artesanal (ver Silverman y Proulx 2002; Vaughn 2009), lo que tendría importantes implicaciones para nuestra comprensión de la utilización de Mina Primavera.

No queremos dar a entender que Mina Primavera fue la única fuente de hematita en la región de Nasca. En nuestra prospección de la región hemos encontrado fuentes adicionales de hematita que no fueron explotadas por los Nasca ni otros mineros prehispánicos. Además, culturas prehispánicas que han explotado minas hace tiempo, ya desaparecieron del registro arqueológico. Creemos, sin embargo, que nuestro trabajo ha brindado nuevas luces sobre la actividad minera prehispánica, todavía poco conocida, y esperamos hacer frente a las preguntas que hemos planteado en la investigación futura.

Agradecimientos: Nos gustaría agradecer a la National Geographic Society por los fondos para completar este proyecto. También nos gustaría agradecer al INC de Lima e Ica por el permiso de excavar en la Mina Primavera. Especialmente agradecemos a Rubén García y Susana Arce. Agradecemos a José Sereveleón, Verity Whalen, Stefanie Bautista, Jessica Kaplan, Meghan Tierney, Yoshiko Tsuji, John Kantner y Cynthia Hotujec por su ayuda en el campo, y Stefanie Bautista y Marcela Poirier por su ayuda en aspectos de la traducción.

\section{Referencias Citadas}

Abbott, M.B. y A.P. Wolfe 2003. Intensive pre-Incan metallurgy recorded by lake sediments from the Bolivian Andes. Science 301:1893-1895.

Aldunate, C., V. Castro y V. Varela 2008. San Bartolo y Cobija: Testimonios de un modo de vida minero en las tierras altas y la costa de Atacama. Estudios Atacameños 35:97-118.

Ballard, C. y G. Banks 2003. Resource wars: The anthropology of mining. Annual Review of Anthropology 32:287-313.

Bakewell, P.J. 1984. Miners of the Red Mountain: Indian Labor in Potosí 1545-1650. University of New Mexico Press, Albuquerque.

Barba, A.A. 1923. El Arte de los Metales. J. Wiley, New York.

Berthelot, J. 1986. The extraction of precious metals at the time of the Inka. En Anthropological History of Andean Polities, editado by J.V. Murra, N. Wachtel y J. Revel, pp. 68-88. Cambridge University Press, Cambridge.
Bird, J.B. 1979. The "Copper Man": A prehistoric miner and his tools from northern Chile. En Pre-Columbian Metallurgy of South America, editado por E.P. Benson, pp. 105-132. Dumbarton Oaks, Washington DC.

Bonavia, D. 1959. Una pintura mural de Pañamarca, Valle de Nepeña. Arqueológicas 5. Publicación del Instituto de Investigaciones Antropológicas, Museo Nacional de Antropología y Arqueología, Lima.

- - - 1985. Mural Painting in Ancient Peru. University of Indiana Press, Bloomington.

Brewer, M.J. 2003. From Myth to Reality: Performing the Devil and Pachamama in the Carnival of Humahuaca. $\mathrm{PhD}$ thesis, Department of Anthropology, University of Chicago.

Burger, R.L. y R. Matos Mendieta 2002. Atalla: A center on the periphery of the Chavin Horizon. Latin American Antiquity 13:153-177. 
Cobo, B. 1890-1895. Historia del Nuevo Mundo. 4 vols. Sociedade de Bibliofilos Andaluces, Sevilla.

Dawson, L.E. 1964. Slip casting: A ceramic technique invented in ancient Peru. Nawpa Pacha 2:107-112.

Eerkens, J.W., K.J. Vaughn y M. Linares Grados 2009. Prehispanic mining in the Southern Nasca Region, Peru. Antiquity 83:738-750.

Eerkens, J.W., K.J. Vaughn, G. Barford y P.R. Williams, s.f. Using iron isotope ratios to source hematite in the Nasca region. Manuscrito sometido a Archaeological and Anthropological Sciences.

Fuller, D.R., 2004. The production of copper in 6th century Chile's Chuquicamata mine, JOM 56, 62-66.

Gisbert, T., 2010. El cerro de Potosí y el dios Pachacámac, Chungará 42, 169-180.

Haeberli, J. 1979. Twelve Nasca panpipes: A study. Ethnomusicology 23:57-74.

Hanks, B. y R. Doonan 2009. From scale to practice: A new agenda for the study of Early Metallurgy on the Eurasian Steppe. Journal of World Prehistory 22:329-356.

Kroeber, A.L. y D. Collier 1998. The Archaeology and Pottery of Nazca, Peru : Alfred L. Kroeber's 1926 Expedition. AltaMira Press, Walnut Creek.

Maldonado, B. y T. Rehren. 2009. Early copper smelting at Itziparatzico, Mexico, Journal of Archaeological Science 36, 1998-2006.

Menzel, D. 1964. Style and time in the Middle Horizon. Nawpa Pacha 2:1-106.

Montoya, M., W. García y J. Caidas 1994. Geología de los Cuadrángulos de Lomitas, Palpa, Nasca y Puquio. INGGEMET (Instituto Geológico Minero y Metalúrgico), Lima.

Nash, J. 1972. Devil in Bolivia's nationalized tin mines. Science and Society 36:221-233.

- - - 1979. We Eat the Mines and the Mines Eat Us: Dependency and Exploitation in Bolivian Tin Mines. Columbia University Press, New York.

Nerantzis, N., 2009. Pillars of power: silver and steel of the Ottoman Empire, Mediterranean Archaeology and Archaeometry 9, 71-85.

Núñez, L. 2006. La orientación minero-metalúrgica de la producción atacameña y sus relaciones fronterizas. En Esferas de Interacción Prehistóricas y Fronteras Nacionales Modernas: los Andes Surcentrales, editado por H. Lechtman, pp. 205-260. IEP-IAR, Lima.

Orefici, G. y A. Drusini 2003. Nasca: Hipótesis y Evidencias de su Desarrollo Cultural. Centro Italiano Studi E Ricerche Archeologiche Precolombiane, Brescia.

Paulsen, A.C. 1974. The thorny oyster and the voice of God: Spondylus and Strombus in Andean prehistory. American Antiquity 39:597-607.

Petersen, G. 1970. Minería y Metalurgia en el Antiguo Perú. Arqueológicas 12:1-140.

- - - 2010. Mining and Metallurgy in Ancient Perú. Special Paper 467. The Geological Society of America, Boulder.
Phipps, E.J.S. 1989. Cahuachi Textiles in the W. D. Strong Collection: Cultural Transition in the Nasca Valley, Peru. Ph.D. dissertation, Department of Art History, Columbia University.

Reimer, P.J., M.G.L. Baillie, E. Bard, A. Bayliss, J.W. Beck, P.G. Blackwell, C. Bronk Ramsey, C.E. Buck, G.S. Burr, R.L. Edwards, M. Friedrich, P.M. Grootes, T.P. Guilderson, I. Hajdas, T.J. Heaton, A.G. Hogg, K.A. Hughen, K.F. Kaiser, B. Kromer, F.G. McCormac, S.W. Manning, R.W. Reimer, D.A. Richards, J.R. Southon, S. Talamo, C.S.M. Turney, J. van der Plicht y C.E. Weyhenmeyer 2009. INTCAL09 and MARINE09 radiocarbon age calibration curves, 0-50,000 years cal bp. Radiocarbon 51:1111-1150

Proulx, D.A., 2006. A Sourcebook of Nasca Ceramic Iconography: Reading a Culture through its Art, Iowa University Press, Iowa City.

Reindel, M. 2009. Life at the edge of the desert: Archaeological reconstruction of the settlement history in the valleys of Palpa, Peru. En New Technologies for Archaeology, editado por M. Reindel y G.A. Wagner, pp. 439-461. Springer, Berlin.

Salazar, D., V. Castro, J. Michelow, H. Salinas, V. Figueroa y B. Mille 2010. Minería y metalurgia en la costa arrecia de la región de Antofagasta, norte de Chile. Boletín del Museo Chileno de Arte Precolombino 15:9-23.

Salazar, D., D. Jackson, J. L. Guendon, H. Salinas, D. Morata, V. Figueroa, G. Manríquez y V. Castro 2011. Early evidence (ca. $12,000 \mathrm{BP}$ ) for iron oxide mining on the Pacific coast of South America. Current Anthropology 52(3):463-475.

Salazar Soler, C. 2006. Supay Muqui Dios del Socavón. Vida y Mentalidades Mineras. Fondo Editorial del Congreso del Perú, Lima.

Schreiber, K. 2005a. Sacred landscapes and imperial ideologies: The Wari empire in Sondondo, Peru. En The Foundations of Power in the Prehispanic Andes, editado por K.J. Vaughn, D.E. Ogburn y C.A. Conlee, pp. 131-150. Archeological Papers of the AAA, Washington D.C.

- - - 2005b. Imperial agendas and local agency: Wari colonial strategies. En The Archaeology of Colonial Encounters: Comparative Perspectives, editado por G.J. Stein, pp. 237-262. School of American Research Press, Santa Fe.

Schreiber, K. y J. Lancho Rojas 2006. Aguas en el Desierto: Los Puquios de Nasca. Fondo Editorial, Pontificia Universidad Católica del Perú, Lima.

Shimada, I. 1994. Pre-Hispanic metallurgy and mining in the Andes: recent advances and future tasks. En In Quest of Mineral Wealth: Aboriginal and Colonial Mining and Metallurgy in Spanish America, pp. 37-73. vol. 33. Geoscience Publications, Louisiana State University Series: Geoscience and Man.

Silverman, H. y D.A. Proulx 2002. The Nasca. Blackwell Publishers, Malden.

Stöllner, T. 2009. Gold in southern Peru? Perspectives of research into mining archaeology. En New Technologies for Archaeology: Multidisciplinary Investigations in Palpa and Nasca, Peru, editado por M. Reindel y G.A. Wagner, pp. 393407. Springer, Berlin.

Taussig, M.T. 1980. The Devil and Commodity Fetishism in South America. University of North Carolina Press, Chapel Hill. 
Unkel, I., A. Kadereit, B. Maechtle, B. Eitel, B. Kromer, G. Wagner y L. Wacker 2007. Dating methods and geomorphic evidence of palaeoenvironmental changes at the eastern margin of the South Peruvian coastal desert (14 degrees 30, S) before and during the Little Ice Age. Quaternary International 175:3-28.

Vaughn, K.J. 2009. The Ancient Andean Village: Marcaya in Prehispanic Nasca. University of Arizona Press, Tucson.

- - - 2010. Emergent leadership in middle-range societies: an example from Nasca, Peru. In The Evolution of Leadership: Transitions in Decision Making from Small-Scale to MiddleRange Societies, editado por K.J. Vaughn, J.W. Eerkens y J. Kantner, pp. 147-168. School for Advanced Research Press, Santa Fe.

Vaughn, K.J., C.A. Conlee, H. Neff y K. Schreiber 2006. Ceramic production in ancient Nasca: Provenance analysis of pottery from the Early Nasca and Tiza cultures through INAA. Journal of Archaeological Science 33:681-689.

Vaughn, K.J., J.W. Eerkens, M. Linares Grados y M.J. Edwards 2007. Hematite mining in the ancient Americas:
Mina Primavera, a 2,000 year old Peruvian mine. Journal of Management 59:21-25.

Vaughn, K.J. y H. Neff 2004. Tracing the Clay Source of Nasca Polychrome Pottery: Results from a Preliminary Raw Material Survey. Journal of Archaeological Science 31:1577-1586.

Vaughn, K.J., H. Neff, C.A. Conlee y K.J. Schreiber 2005. A compositional analysis of Nasca polychrome pigments: Implications for craft production on the prehispanic south coast of Peru. En Laser Ablation ICP-MS: A New Frontier in Archaeological Characterization Studies, editado por R.J. Speakman y H. Neff, pp. 138-154. University of New Mexico Press, Albuquerque.

Vaughn, K.J., H. van Gijseghem, J.W. Eerkens y M.D. Linares Grados 2010. Two millennia of Prehispanic mining in Nasca, Peru. Ponencia presentada en la Primera Reunión Internacional sobre Minería Prehispánica en América, Taltal-San Pedro de Atacama, Chile.

Yacovleff, E. y J. C. Muelle 1934. Notas al trabajo colorantes de Paracas. Revista del Museo Nacional 3:154-168.

\section{Nota}

1 Tenga en cuenta que se había propuesto originalmente que estas herramientas fueron hechas de sílex y basalto (Vaughn et al. 2007:17), sino en un análisis adicional en el año 2010 hemos corregido nuestra evaluación preliminar. 